\title{
Protéger nos patients âgés contre les réactions indésirables aux médicaments
}

par Glen Brown

$\mathrm{L}$ 'expérience en soins pharmaceutiques et les résultats apparaissant dans la littérature ont montré que les patients âgés courent plus de risque de subir des réactions indésirables aux médicaments (RIM) que les populations de patients plus jeunes. Dans le présent numéro, Mihajlovic et collab. ${ }^{1}$ ajoutent à ce savoir grâce à une analyse systématique des caractéristiques individuelles qui semblent rendre les patients plus susceptibles à subir des RIM. Leur objectif était d'aider les pharmaciens à discerner les patients présentant un risque plus élevé de RIM au sein d'une population générale recevant des soins de pharmaciens. Ainsi, les pharmaciens pourraient mieux orienter leurs activités là où elles auraient potentiellement le meilleur effet. Mais alors, comment utiliser cette information lorsqu'on procède au triage d'un important nombre de cas?

OAu cours des cinq dernières années, plusieurs groupes de chercheurs ont tenté de créer, de valider et de mettre en place des mécanismes servant à repérer les patients âgés qui profiteraient le plus d'interventions visant à réduire les risques de RIM. La stratégie la plus reconnue emploie les critères de Beers qui reposent sur une liste de médicaments présentant un risque élevé d'être inappropriés pour les patients âgés ${ }^{2}$. À titre d'exemple, des pharmaciens du Québec ont montré qu'une vérification automatisée des dossiers médicaux de patients à l'aide de certains critères de Beers permettait de dépister les patients à qui l'on avait prescrit des médicaments inappropriés ${ }^{3}$. Dans cette étude, le dépistage informatisé a permis aux pharmaciens d'identifier efficacement les patients à risque de RIM et d'intervenir pour modifier leur traitement.

On a montré que les pharmaciens exerçant aux services des urgences ont freiné la prescription de traitements inappropriés, selon les critères de Beers, de trois façons : en éduquant les prescripteurs; en rédigeant des mises en garde sur des médicaments à éviter selon le patient, mises en garde que l'on peut mettre en place par le biais de la saisie électronique des ordonnances par les prescripteurs; et en offrant de la rétroaction aux prescripteurs quant à leur performance ${ }^{4}$. Ces étapes s'apparentent aux techniques de rétroaction et d'intervention utilisées par les programmes de gérance des antimicrobiens. Dans un précédent numéro du Journal canadien de la pharmacie hospitalière, Slaney et collab. ${ }^{5}$ ont montré que l'utilisation des critères de Beers permettait de repérer les patients âgés ayant besoin d'un autre niveau de soins (soins de longue durée) qui avaient par la suite subi une RIM. D’après ces résultats, la vérification des dossiers médicaux afin d'y relever la présence de médicaments inappropriés, selon les critères de Beers, aiderait le pharmacien à discerner les patients âgés requérant le plus son attention.

D'autres chercheurs ont utilisé les critères STOPP/START (Screening Tool of Older Person's Prescriptions/Screening Tool to Alert doctors to Right Treatment: des outils de dépistage des médicaments inappropriés en gériatrie), fondés sur des données probantes, pour déceler les patients ayant besoin d'une évaluation de leur pharmacothérapie ${ }^{6}$. Ces outils contiennent des listes de 81 scénarios combinant médicament, maladie et patient qui devraient mener à considérer l'interruption d'un traitement prescrit et de 34 scénarios combinant médicament, maladie et patient dans lesquels devrait être envisagée l'amorce d'une pharmacothérapie. Bien que ce processus puisse sembler complexe et laborieux, l'ensemble des scénarios recommandés sont faciles à comprendre et à identifier pour les pharmaciens en exercice. On a montré que l'utilisation des critères de dépistage STOPP/ START accroît la pertinence de la prescription ${ }^{7}$ et réduit le taux de réadmissions liées aux médicaments ${ }^{8}$. Des chercheurs de la Nouvelle-Écosse ont découvert que les outils de dépistage STOPP/START étaient potentiellement plus efficaces que les critères de Beers pour déceler des pharmacothérapies qui risqueraient de causer une RIM chez les patients âgés. Ils ont aussi observé que leur utilisation pourrait avoir des avantages mesurables en ce qui concerne le bien-être ultérieur des patients et le recours aux ressources en santé ${ }^{9,10}$. 
D'autres chercheurs ont montré que les pharmaciens peuvent améliorer les pratiques de prescription ${ }^{11}$ et réduire le nombre de RIM subséquentes ${ }^{12}$ lorsqu'ils emploient une méthode structurée pour détecter les patients et les interroger dans le but d'évaluer leur pharmacothérapie. Dans ces études, les pharmaciens utilisaient un logiciel d'aide à la décision qui employait les outils d'évaluation décrits ci-dessus, l'évaluation des données actuelles de laboratoire et la comparaison des médicaments pris antérieurement et actuellement par les patients. Ils ont observé que cette méthode permettait d'éviter une RIM pour chaque 14 patients évalués ${ }^{12}$. L'emploi d'un indice de fragilité servant à identifier les personnes âgées qui courent un plus grand risque de subir des RIM pourrait aussi aider le pharmacien pendant le processus de triage ${ }^{13}$. Un simple score d'évaluation s'appuyant sur seulement cinq variables cliniques (pharmacothérapie de plus de 8 médicaments, hyperlipidémie, leucocytose, emploi d'antidiabétiques et séjour de plus de 12 jours à l'hôpital) a été validé pour prédire quels patients très âgés (c'est-à-dire de plus de 80 ans) sont susceptibles de subir une RIM $^{14}$.

Les recherches qui montrent que nos patients âgés sont plus à risque de subir des RIM (dont l'étude par Mihajlovic et collab. ${ }^{1}$ ) ainsi que les avantages établis des techniques de dépistage et d'intervention décrites ci-dessus devraient inciter tous les pharmaciens d'établissements du Canada à mettre en place des méthodes qui permettent d'identifier ces patients vulnérables pour ensuite intervenir. Chaque pharmacien et, plus particulièrement, les gestionnaires en pharmacie clinique devraient passer en revue l'organisation de leurs services ou de leurs programmes de soins pharmaceutiques afin d'évaluer comment les techniques de dépistage peuvent aider à repérer les patients âgés méritant plus d'attention de la part du pharmacien que la population générale. Lutilisation des technologies de l'information (notamment les systèmes de distribution, les systèmes d'information sur les patients et les dossiers de santé informatisés) dans le but de détecter les patients à risque, en s'appuyant sur des critères aux avantages éprouvés, aurait la portée la plus importante à moindre coût en ce qui a trait au temps des pharmaciens. Nos patients âgés ont besoin de ces soins... il est temps d'agir!

[Traduction par l'éditeur]

\section{Références}

1. Mihajlovic S, Gauthier J, MacDonald E. Patient characteristics associated with adverse drug events in hospital: an overview of reviews. Can J Hosp Pharm. 2016;69(4):294-300.

2. American Geriatric Society 2012 Beers Criteria Update Expert Panel. American Geriatric Society updated Beers criteria for potentially inappropriate medication use in older adults. J Am Geriatr Soc. 2012;60(4):616-31.

3. Arvisais K, Bergeron-Wolff S, Bouffard C, Michaud AS, Bergeron J, Mallet $\mathrm{L}$, et al. A pharmacist-physician intervention model using a computerized alert system to reduce high-risk medication use in elderly inpatients. Drugs Ageing. 2015;32(8):663-70.
4. Moss JM, Bryan WE 3rd, Wilkerson LM, Jackson GL, Owenby RK, Van Houtven C, et al. Impact of clinical pharmacy specialists on the design and implementation of a quality improvement initiative to decrease inappropriate medications in a Veterans Affairs emergency department. J Manag Care Spec Pharm. 2016;22(1):74-80.

5. Slaney H, MacAulay S, Irvine-Meek J, Murray J. Application of the Beers criteria to alternate level of care patients in hospital inpatient units. Can J Hosp Pharm. 2015;68(3):218-25.

6. O'Mahony D, O'Sullivan D, Byrne S, O'Connor MN, Ryan C, Gallagher P. STOPP/START criteria for potentially inappropriate prescribing in older people: version 2. Age Ageing. 2015;44(2):213-8.

7. Gallagher PF, O'Connor MN, O'Mahony D. Prevention of potentially inappropriate prescribing for elderly patients: a randomized controlled trial using STOPP/START criteria. Clin Pharmacol Ther. 2011;89(6):845-54.

8. Gillespie U, Alassaad A, Hammarlund-Udenaes M, Mörlin C, Henrohn D, Bertilsson $\mathrm{M}$, et al. Effects of pharmacists' interventions on appropriateness of prescribing and evaluation of the instruments' (MAI, STOPP and STARTs') ability to predict hospitalization—analyses from a randomized controlled trial. PLoS One. 2013;8(5):e62401.

9. Hill-Taylor B, Sketris I, Hayden J, Byrne S, O'Sullivan D, Christie R. Application of the STOPP/START criteria: a systematic review of the prevalence of potentially inappropriate prescribing in older adults, and evidence of clinical, humanistic and economic impact. J Clin Pharm Ther. 2013;38(5):360-72.

10. Hill-Taylor B, Walsh KA, Stewart S, Hayden J, Byrne S, Sketris IS. Effectiveness of the STOPP/START (Screening Tool of Older Persons' potentially inappropriate Prescribing/Screening Tool to Alert doctors to the Right Treatment) criteria: systematic review and meta-analysis of randomized controlled studies. J Clin Pharm Ther. 2016;41(2):158-69.

11. O'Sullivan D, O'Mahony D, O'Connor MN, Gallagher P, Cullinan S, O'Sullivan R, et al. The impact of a structured pharmacist intervention on the appropriateness of prescribing in older hospitalized patients. Drugs Aging. 2014;31(6):471-81.

12. O'Sullivan D, O'Mahony D, O'Connor MN, Gallagher P, Gallagher J, Cullinan S, et al. Prevention of adverse drug reactions in hospitalised older patients using a software-supported structured pharmacist intervention: a cluster randomized controlled trial. Drugs Aging. 2016;33(1):63-73.

13. Cullinan S, O'Mahony D, O'Sullivan D, Byrne S. Use of a frailty index to identify potentially inappropriate prescribing and adverse drug reaction risks in older patients. Age Ageing. 2016;45(1):115-20.

14. Tangiisuran B, Scutt G, Stevenson J, Wright J, Onder G, Petrovic M, et al. Development and validation of a risk model for predicting adverse drug reactions in older people during hospital stay: Brighton Adverse Drug Reactions Risk (BADRI) Model. PloS One. 2014;9(10):e111254.

Glen Brown, Pharm. D, FCSHP, BCPS (QA), BCCCP, est membre du Service de pharmacie du St Paul's Hospital de Vancouver, en ColombieBritannique.

Intérêts concurrents : Aucun déclaré.

\section{Adresse de correspondance :}

Dr Glen Brown

Pharmacy

St Paul's Hospital

1081 Burrard Street

Vancouver (C.-B.) V6Z 1Y6

Courriel : gbrown@providencehealth.bc.ca 